\title{
Contrast, Noise, and Blur Affect Performance and Appreciation of Digital Radiographs
}

\author{
Ineke M.C.J. van Overveld
}

\begin{abstract}
We have studied the effect of the simultaneous variation of contrast, noise, and blur on the perceived quality of digital radiograph images. Three images were processed with different levels of roentgen photon noise, different luminance (gray-level) ranges, and different amounts of Gaussian blur. Observers had to rate the quality of the images on a numerical scale. To study how the quality judgement is related to the visibility of small details (diagnostic quality), an artificial detail was added to each image. The detail could be in two possible locations. The visibility of this detail was measured in a two-alternative forced-choice experiment. One of the conclusions is that the quality judgment was based mainly on blur, whereas the visibility of details was affected mostly by noise.

Copyright 1995 by W.B. Saunders Company
\end{abstract}

KEY WORDS: image quality, performance, detection, appreciation, noise, contrast, sharpness.

$I^{\mathrm{N}}$ DESIGNING or using a medical imaging device, the aim is to obtain the best possible image quality given the restrictions of the device. But how are we to measure the quality of such images? First of all, we can distinguish between objective and subjective quality measurements. Among the objective measures are signal-to-noise ratio, modulation-transfer function (MTF) and information content measures. ${ }^{1-3}$ Subjective measurements are used to investigate how humans perceive quality. Here it is usually the performance-oriented quality that is studied, ie, the degree of usefulness of an image for its intended purpose, where this purpose can be defined at different levels (effect on diagnosis, treatment, or morbidity and mortality). ${ }^{1}$ This type of quality is most often measured in experiments in which specific details have to be detected, in either clinical or phantom images. Contrast-detail analyses and receiver operating characteristic-type studies ${ }^{4}$ are examples of this.

Little work has been done on a different type

From the Institute for Perception Research (IPO), Eindhoven, The Netherlands.

Address reprint requests to W.M.C.J. van Overveld, PhD, Institute for Perception Research/IPO, PO Box 513, $5600 \mathrm{MB}$ Eindhoven, The Netherlands.

Copyright 101995 by W.B. Saunders Company

0897-1889/95/0804-0006\$3.00/0 of quality, namely the appreciation-oriented or cosmetic quality, although it has been noted ${ }^{5}$ that this may play an important role even when images are used solely for diagnosis. Recently, some studies ${ }^{6,7}$ have concentrated on this appreciation-oriented quality. These studies used numerical category scaling to determine the perceived quality of digital angiograms as a function of certain physical characteristics of the images. This work may be compared with TV image quality studies. ${ }^{8,9}$

However, very few investigators appear to have studied the combination of the two types of quality. An example can be found in the work of MacMahon et $a l,{ }^{10}$ who asked the subject about the ease of reading for thorax images. More recently, Cosman et al ${ }^{11}$ studied the comparison between subjective and objective measurements for compressed computed tomography and magnetic resonance images. In the nonmedical field, Roufs and Boschman ${ }^{12}$ compared comfort ratings with performance measures such as the reading speed and search velocity for text displayed on a visual display unit. In each of these cases it was found that subjective and objective quality could behave differently when physical parameters in the stimuli were varied. The research reported here concentrates on a combination of appreciationand performance-oriented quality for digital radiograph angiograms.

One of the reasons for studying how these two types of quality behave and interact was the following. In previous studies ${ }^{6,13,14}$ it was found that subjects used details in images to judge the quality; when a certain blood vessel was visible, they considered the quality better than when that vessel was not visible. Thus performanceoriented quality (detection of a vessel) got mixed up with the more general, appreciationoriented type of quality. In the study reported here, an experiment was designed in which subjects had not only to judge the overall quality as before, but also to detect small details in the images. Thus, the types of quality could be separated and it was possible to investigate whether they are really one and the same thing 
or whether they behave differently when various aspects of images are changed. Three parameters were varied in the images: noise, contrast, and blur. According to Kundel ${ }^{5}$ and Van Overveld, ${ }^{13,14}$ these three parameters are the most important ones when radiologists have to judge the quality of radiograph images.

\section{MATERIALS AND METHODS}

Images. Three 1,024- $\times$ 1,024-pixel black-and-white images were used. They were angiographic (Philips Medical Systems Integris, Best, The Netherlands) images taken from actual patients. All images were acquired at 10 bits per pixel, but displayed at 8 bits per pixel. We refer to the images as "cer," "kid," and "leg."

Cer is a subtracted (digital subtraction angiography) image of cerebral vessels. Very thin vessels are visible in this image provided the contrast is high enough. The smooth bright background of this image is quite sensitive to noise.

Kid is a contrast image of the artery feeding the kidney. Additionally, the kidney itself is filled with contrast liquid. Both the artery and its ramifications are important, but the contour of the kidney against its background also matters.

Leg is a nonsubtracted view of a leg just below the knee, where the thin blood vessels pass over both the bone and the soft tissue, giving rise to very different types of contrast, all of which are diagnostically important.

These three images are referred to as "scenes." Close-up views of parts of the scenes are shown in Fig 1 (see Added details). The images have been varied as to noise, contrast, and sharpness using the following processing techniques.

Noise variation. In radiograph imaging systems, several sources of noise can be distinguished, such as roentgen ray quantum noise (caused by the quantum nature of the roentgen ray), image intensifier noise (fixed pattern noise caused by inhomogeneities in the image intensifier screen), and electronic camera noise. For our purposes, roentgen ray quantum noise was considered to be the most important noise source. The amount of this noise was varied by computer simulation of the effect of a varying $x$-ray dose (see Fig 2).

A nonlinear function (white compression) is applied to the images before storage and export. Before adding the extra noise to the images the inverted function should be applied to linearize the relationship between pixel values and number of $x$-ray quanta at the input of the detection chain. At this level two types of noise were added: a variable amount of Poisson noise simulating the $x$-ray photon noise and a small fixed amount of Gaussian noise simulating the electronic camera noise. The mean gray value (of the $216-\times 216$-pixel center of the image) at the output of the image intensifier could be related to the $x$-ray dose and, thus, to the number of photons per pixel; from this the standard deviation of the Poisson noise was determined as the square root of the number of photons. The photon noise was filtered through the MTF of the optical system, but the electronic noise was not. It was verified by means of phantom images that this simulation gave effects comparable with those of true $x$-ray dose variation.

The upper limit of the range of noise levels applied in our
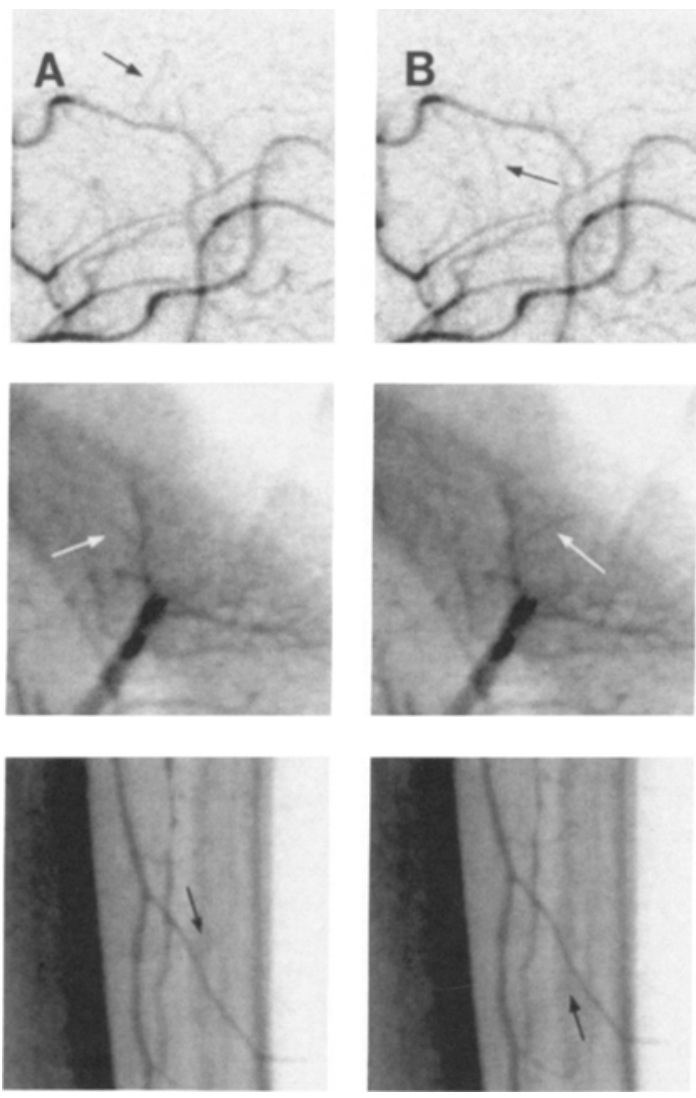

Fig 1. A and B versions of all scenes: enlarged and contrastenhanced areas in which the artificial detail occurs (indicated by arrows). Top row, cer; middle row, kid; bottom row, leg.

experiments was chosen as the threshold of acceptability (as regards diagnostic usefulness). This threshold was established with the aid of two radiologists whose task was to indicate this threshold when presented with a large range of noise levels applied to each of the scenes. The lower limit of the range of noise levels was chosen as the lowest dose that still gave the same impression of noise as the original image,

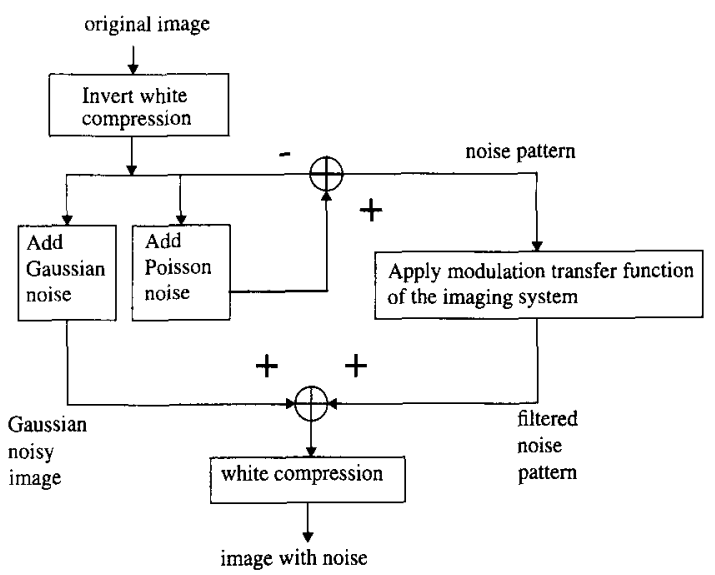

Fig 2. Noise simulation scheme (see Noise variation). 
which was considered to be noise free because of the high dose used during the actual acquisition (around $700 \mu \mathrm{R}$ ). Note that the limits differed from scene to scene. Within the intervals defined by these limits, four logarithmically equidistant noise levels were used in the experiments. The levels are shown in Table 1.

Contrast variation. The contrast was varied as follows. First of all, we selected the smallest possible gray-level range containing all the relevant medical information. We accordingly refer to this as the relevant range. This range was, of course, different for each of the scenes because it was based on the gray-value histograms of the scenes. The ranges are given in the top row of Table 2 . To vary the contrast in an image, the relevant range was mapped to ranges of different widths (see below). Values outside the relevant range were clipped to the minimum and maximum values of the output range. The resulting output gray values were fed to the hard-copy unit, which applied a look-up table (LUT) for perceptual linearization. ${ }^{15}$ The effect of this LUT is that a step wedge of linearly increasing gray values ought to be perceived as a wedge of linearly increasing brightness. Although this depends on the amount of ambient light and the adaptation level, the LUT approximately creates the desired effect.

The contrast was graded into four levels, corresponding to widths $255,200,150$, and 100 of the output gray-value range. These widths will be referred to as contrast levels. Clearly, 255 is the maximum possible width for 8-bit images. It is obtained by linearly mapping the relevant range to the interval 0-255. For the smaller ranges we are left with a degree of freedom. This was used to keep the average perceived brightness in the images constant over contrast variation because we wanted to study the effect of contrast separately from the effect of brightness. Keeping the average brightness constant is the same as keeping the average gray value constant if we may assume that the hard-copy unit's LUT maps gray values to perceived brightness in the above-mentioned way. The average gray values of the images with a contrast level of 255 were computed from the gray-value histogram. The blanking parts of the images (black surroundings of the circle in which the actual medical data is displayed) were excluded from the histogram computations because the black parts were kept artificially at level 0 when the gray-value ranges were varied. The average gray values in the image circles are given in the bottom row of Table 2. For each of the scenes a number, $x_{k}$, could be found such that, when the original relevant range was linearly scaled to $\left[x_{l}, x_{I}+100\right]$, the average gray value was equal to the average value shown in Table 2 . Similarly, $x_{2}$ and $x_{3}$ existed such that the same average gray values were found after rescaling the relevant range to $\left[x_{2}, x_{2}+150\right]$ or to $\left[x_{3}\right.$,

Table 1. Noise Levels Applied to Cer, Kid, and Leg Scenes

\begin{tabular}{cccc}
\hline \multirow{2}{*}{$\begin{array}{c}\text { Noise } \\
\text { Level }\end{array}$} & \multicolumn{3}{c}{ Photons/Pixel (X-Ray Dosel } \\
\cline { 2 - 4 } & $1,030(370 \mu R)$ & $260(90 \mu R)$ & $130(45 \mu R)$ \\
1 & $410(150 \mu R)$ & $130(45 \mu R)$ & $65(20 \mu R)$ \\
2 & $163(55 \mu R)$ & $65(20 \mu R)$ & $32(10 \mu R)$ \\
3 & $65(20 \mu R)$ & $32(10 \mu R)$ & $16(5 \mu R)$ \\
4 & & & \\
\hline
\end{tabular}

Table 2. Relevant Ranges, Output Gray-Value Ranges and Average Gray Values for Each Contrast Level and Scene

\begin{tabular}{lrrr} 
& \multicolumn{1}{c}{ Cer } & \multicolumn{1}{c}{ Kid } & \multicolumn{1}{c}{ Leg } \\
\hline Relevant range & $85-200$ & $30-170$ & $25-190$ \\
Interval (width 255) & $0-255$ & $0-255$ & $0-255$ \\
Interval (width 200) & $48-248$ & $30-230$ & $25-225$ \\
Interval (width 150) & $92-242$ & $53-203$ & $40-190$ \\
Interval (width 100) & $135-235$ & $78-178$ & $58-158$ \\
Average gray value & 222 & 136 & 99 \\
\hline
\end{tabular}

$x_{3}+200$ ]. The output ranges for each of the widths and the average gray values for each scene are given in Table 2 .

Sharpness variation. After the noise and contrast transformations were applied to the images, they were varied in sharpness. This was done using Gaussian filters of different sizes because this kind of filter produces a good approximation of the type of blurring that actually takes place in an imaging system. The levels of blurring used in the experiment correspond to Gaussian blurring kernels with radii (standard deviation in pixels) of 1.0,2.0, and 3.0. The radius of the blur function is a measure for the amount of unsharpness. The fourth level consisted of no blur, ie, only the original unsharpness in the image (caused by the acquisition chain and the printer) was present. This was negligible compared with the extra blur. Figure 3 shows the effect of the blurring on the MTF. The graphs show how the modulation of sinusoidal grids is attenuated by the Gaussian blur; they do not define the MTF of the whole imaging chain.

Added details. The processing described in the previous sections was not applied to the original scenes, but to scenes with artificial details added to them. The extra detail was blurred and varied in contrast and noise along with the rest of the image. Thus, the visibility of the detail varied simultaneously with the perceived quality of the stimuli in general

We made two versions of every scene: one in which an extra vessel appeared on one side of an existing vessel, and one where the extra vessel appeared on the other side of the existing vessel. Thus, we had an A and B version of each

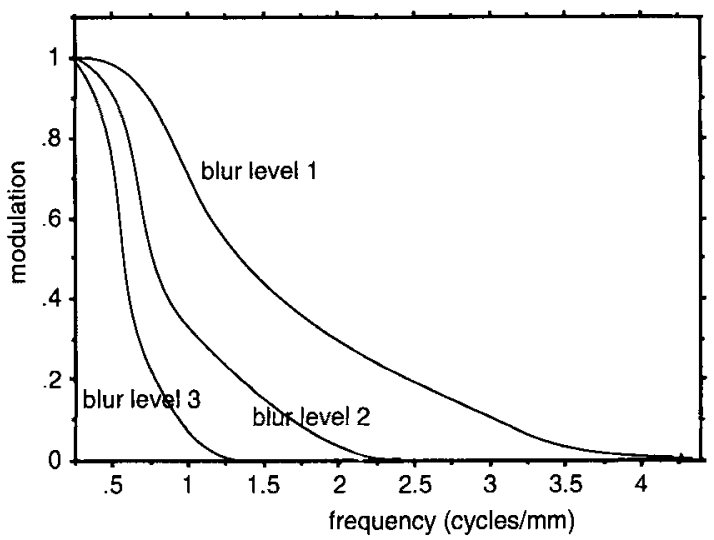

Fig 3. Modulation-transfer characteristics as a consequence of Gaussian blurring at various levels. The frequency is expressed in cycles per millimeter on the printed image. 
scene. Figure 1 shows the enlarged region of the images in which the extra detail occurs, with the A and B versions side by side for each scene. For illustration purposes, the contrast in this figure is much higher than that actually used in the experiments.

The list of all 64 possible (noise, contrast, blur) triples was randomly partitioned into two equal parts: an A part and a $B$ part. The A version of the image was processed with the 32 noise, contrast, and blur combinations occurring in the $A$ part of the list, and the remaining (noise, contrast, blur) combinations were applied to the B version of the scene. A different partitioning of the list was used for each of the three original scenes.

Because we wanted to determine how the contrast affected the detectability of the added details, the gray values in the immediate neighborhood of the vessel were kept more or less constant in gray value, so that only the contrast of the vessel with its background varied when the overall contrast level was varied. The brightness of the region around the vessel was fixed because variation of the brightness as well as the contrast would have complicated interpretation of the results. As a consequence of this decision, the extra detail and its background had to have approximately the same gray value as the average gray value of the whole image. This follows from the construction of gray-level ranges as already explained.

Figure 4 shows how the gray values vary in the neighborhood of the added detail for the cer scene. The figure shows different configurations of contrast, noise, and blur levels. Of course, there is no straight border between the gray values of the vessel and its background; even if there was no noise at all (which there is, even at the lowest noise level), the image shows the two-dimensional projection of a round object, which, by itself, ensures a smooth transition from the minimum intensity (maximum density) at the center of the vessel to the maximum intensity in the background. It should be noted that the artificial vessel was created by copying and pasting an existing real vessel in the same image in a new location, so that it does indeed have the same gray-level distribution as a real vessel. The ragged appearance of the curve in Fig $4 \mathrm{~A}$ is caused by the $\mathrm{x}$-ray noise that was present in the original image and that was enhanced by the contrast stretching.

Experimental set-up for experiment 1 . The stimuli for the first experiment were produced as described in the previous sections. First, the extra detail was added, then the noise was added, then the contrast was varied, and finally the images were blurred. This order of processing mimics a real radiograph imaging system, where the quantum noise is produced before the $x$-rays enter the image intensifier and the contrast and blur variations occur after this as a consequence of image intensifier, camera, and display characteristics. The images were printed on a laser hardcopy unit using a LUT for perceptual linearization.

The images were put on a view box with a luminance of 2,500 candela $/ \mathrm{m}^{2}$. To simulate the average amount of ambient light to be found in reading-rooms, a desk lamp was used which lit the wall behind the view box. The wall reflected about $70 \mathrm{~cd} / \mathrm{m}^{2}$, and $1 \mathrm{~cd} / \mathrm{m}^{2}$ was reflected from the front of the view box as a result of this. There were no uncovered parts of the view box. The viewing distance was $50 \mathrm{~cm}$ and the radius of the images was $12 \mathrm{~cm}$ (viewing angle: 13 degrees). Nine subjects took part in the experiment. They were not familiar with radiograph images and are accordingly referred to as nonexperts. All subjects had normal or corrected-to-normal vision (visual acuity was at least 1.2). The subjects could inspect the images freely, without restrictions in pupil size or fixation point.

The subjects' first task was to judge the quality of each stimulus by assigning it an integer number between 1 and 10. The subject was instructed to take both diagnostic and cosmetic considerations into account. A score of 10 corresponded to the highest quality and 1 , to the lowest. The 64 images of a single scene were shown on the view box in randomized groups of 16 at a time. Every image was judged once by each subject. In a second session, subjects had to
Fig 4. Profile of the artificial vessel in the cer image. The graph shows the gray values of all pixels on a path crossing the vessel. The intersection point of the path and the vessel lies approximately in the middle of the vessel, and the path runs perpendicular to the vessel. The distance on the $X$-axis of the graph is measured along the path, where 0 indicates the intersection point. a through d show different levels of parameters, as indicated in the plot. a corresponds to the optimum setting and $d$, to the worst possible setting. The three different curves in each plot show the profiles of three different paths, shifted by one pixel toward the $Y$-axis.
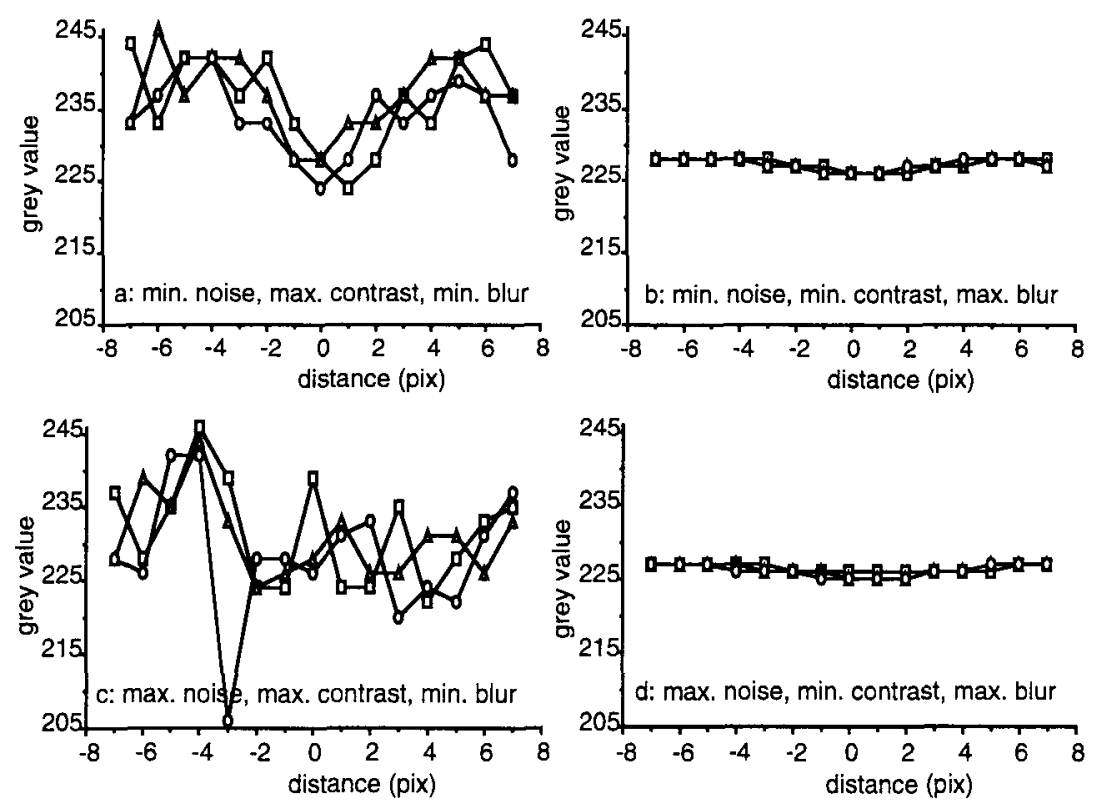
detect the location of the added detail. A two-alternative forced-choice (2AFC) paradigm was used; when the subject was not certain in which of the two locations the vessel appeared, he or she had to guess. First, the subject was shown the two possible locations of the detail, using two obvious examples. After this, every image had to be judged five times in pseudorandom order. There were enough different images between two occurrences of the same stimulus to preclude memory effects. No time constraints were imposed on the subjects, but both the quality rating and the detection task took only a few seconds per image. The experiment consisted of four sessions and it took 2.5 hours in all.

Experimental set-up for experiment 2. We wanted to compare the results of experiment 1 with those of radiologists and those of a group that is more or less in between radiologists and nonexperts in experience, namely the application specialists. Quality judgements elicited by these groups had been compared previously ${ }^{6.7}$ for different variations in radiograph images. Because the observers' time could not be disposed of as freely as in experiment 1 , the experiment was shortened to 30 minutes by taking the following measures: (1) Only two scenes were used instead of three. The leg scene was omitted for reasons that will be explained below. (2) All parameters were varied with only three levels instead of four. This was done by omitting level 150 for the contrast, level 2 for the noise (see Table 1), and level 1 for the blur. Careful analysis of the results showed that the results for these levels were usually close to those of one of the other levels, thus allowing us to interpolate the results for the three levels to arrive at the results for the fourth level. (3) The task of the detection part was altered. Instead of repeating the $2 \mathrm{AFC}$ task five times per stimulus, subjects were asked for a confidence rating when they gave their response. This means that, instead of just " $A$ " or " $B$," the possible answers were: "A, sure," "A, not sure," "?" (don't know), "B, not sure" or "B, sure." Only two replications per stimulus were used with this approach.

The confidence rating gives extra information in the sense that it tells us whether a subject feels comfortable with the visibility of the details; if a radiologist was not sure whether he saw a detail, this might influence his diagnosis, even though he might guess the correct location in $100 \%$ of the trials if he were forced to give a binary answer. This is the reason for omitting the leg scene from this experiment; in a pilot experiment, it was found that subjects felt more sure with the $A$ version than with the $B$ version of this scene, so that there was a bias in the response.

Because detection with confidence rating is not completely equal to a $2 \mathrm{AFC}$ experiment, it was checked whether the two approaches gave similar results. This was done by comparing the results of one subject who participated in both experiments 1 and 2. The correlation between the results of this subject for experiments 1 and 2 was of the same order of magnitude as the correlation between results of different subjects within either experiment 1 or experiment 2.

In experiment 2, four nonexperts (none of whom had participated in experiment 1), four application specialists, and four radiologists participated as observers. The viewing circumstances for the subjects varied somewhat, depending
Table 3. ANOVA Significant Factors for Experiment 1

\begin{tabular}{lc}
\hline \multicolumn{1}{c}{ Factor } & \% Explained Variance \\
\hline Scene & 30.8 \\
Scene/noise & 16.9 \\
Noise & 12.5 \\
Contrast/blur & 4.5 \\
Error & 10.3 \\
\hline
\end{tabular}

The dependent variable is percentage correct.

on the view boxes and illumination available at the sites where the experiment was performed. As a rule, the situation for the nonexperts in experiment 1 was copied as closely as possible.

\section{RESULTS}

Experiment 1 . A quality score and a percentage correct were obtained for each stimulus and subject. Quality scores were submitted to a $z$-transformation to bring all scores into the same range (to compensate for the fact that one observer might use scores from 1 to 6 , whereas another used 5 to 10$).^{7}$ Percentages correct were obtained by dividing the number of correct answers by the maximum possible number of correct scores. Percentages lower than 50\% were changed to $50 \%$ because performance below chance level was not relevant.

Tables 3 and 4 show the results from analysis of variance (ANOVA) computations; Table 3, the results for the percentage of correct detections as dependent variable; and Table 4, those for the z-transformed quality scores. The first column lists the factors and interactions that are significant at the $1 \%$ level and contribute at least $3 \%$ to the variance. The $3 \%$ restriction was imposed because there were quite a few factors accounting for less than $3 \%$ of the total variance, and their inclusion in the tables would only have veiled the more important effects. The second column shows the fraction of variance explained. Because the data was averaged over the subjects in this analysis, there was only one observation per combination of parameters, so that the full interaction term had to be used as the error term. ${ }^{16}$ This is listed in the

Table 4. ANOVA Significant Factors for Experiment 1

\begin{tabular}{cc}
\hline Factor & \% Explained Variance \\
\hline Blur & 60.0 \\
Contrast & 17.5 \\
Noise & 6.2 \\
Error & 2.8 \\
\hline
\end{tabular}

The dependent variable is z-corrected quality. 
Fig 5. Percentage correct for three scenes (left column, cer; middle column, kid; right column, leg). The top and bottom rows show results for the lowest and highest blur levels, respectively. The noise level is indicated on the $X$-axis, where 1 denotes the lowest noise level for that scene and 4 , the highest noise level (see Table 1). The $Y$-axis shows the fraction of correct answers, averaged over the subjects. The dashed line shows the $75 \%$ level, which may be regarded as the detection threshold. The contrast level is encoded in the type of data points: (O), 255; ( $\square), 200 ;(\triangle), 150 ;(\diamond)$. 100.
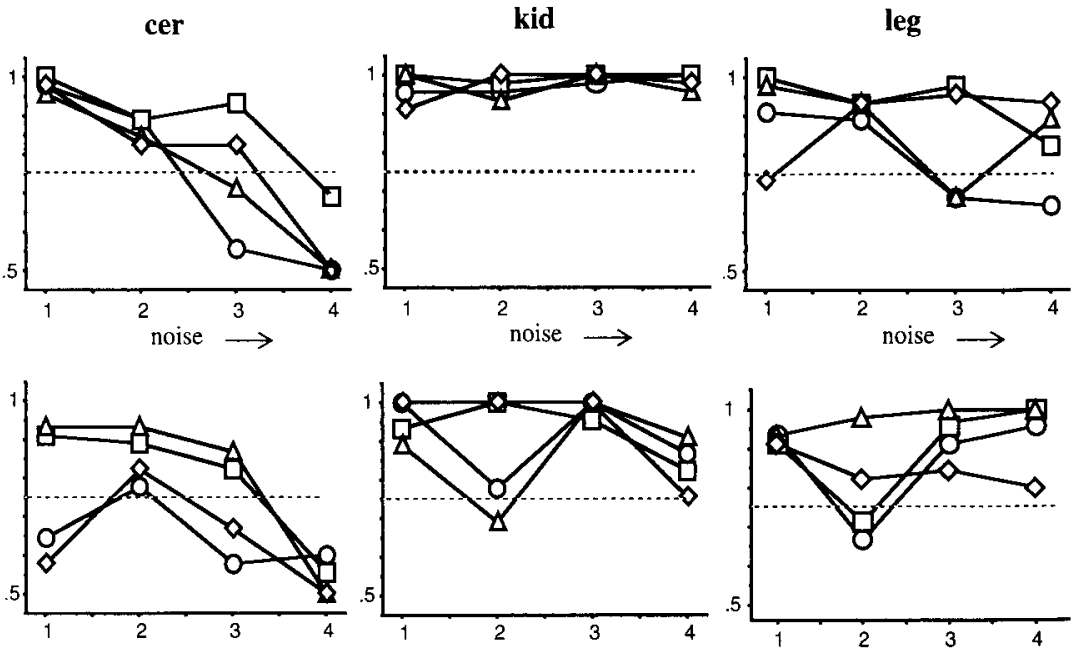

bottom row of the tables. It should be noted that an ANOVA in which the subjects were treated as replications gave the same main effects, in the same order of significance, but the results were much more noisy owing to interobserver variation. For the percentage correct, this error is partly explained by the $2 \mathrm{AFC}$ method, which gives inaccurate measurements even for a single subject.

We can study the nature of the effects and interactions by plotting the data for different combinations of factors (see Figs 5 and 6 ). In the figures, the results are averaged over the subjects. It should be noted that, though there is some interobserver variation, the majority of the results mentioned below still hold for the individual subjects as well as for the average. In Fig 5, the standard error in the mean for (almost) every stimulus point is less than 0.1 . In Fig 6 , the $z$ scores have been linearly trans-
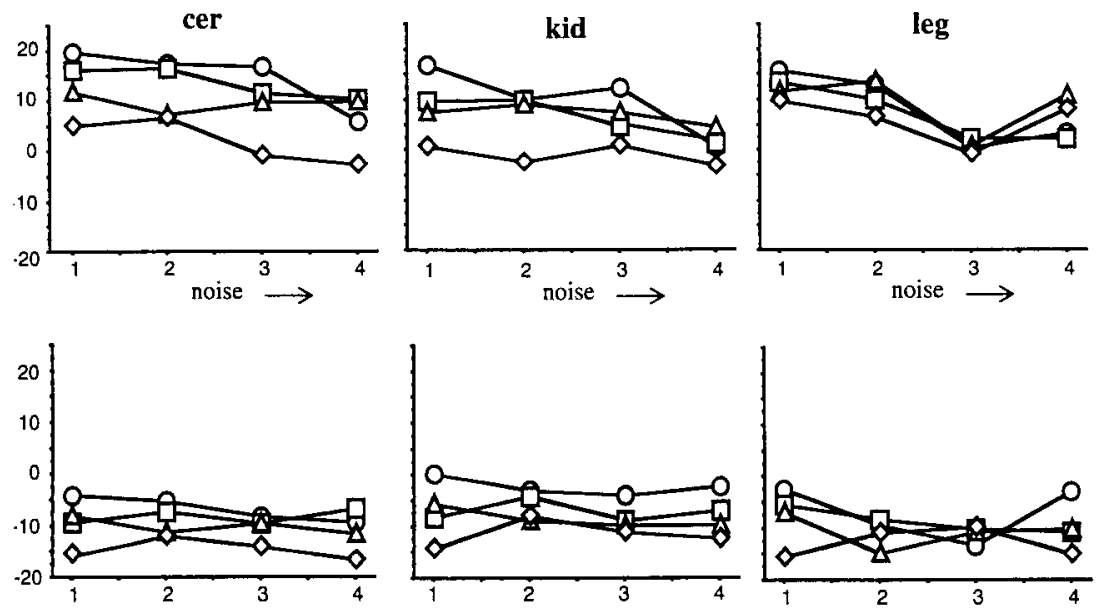

Fig 6. Quality scores for three scenes. The order of the graphs and the labeling of curves and the $X$-axis are the same as in Fig 5 . The $Y$-axis shows the quality scores averaged over the subjects via z-transformation; the unit of the scale equals the SEM. 
found in previous experiments where the contrast parameter gamma was varied, $, 6,7$ because gamma variation has different effects in different parts of the gray-value range. In that case, it matters in which part of the gray-value range the relevant information is contained. Obviously this is scene dependent.

The effect of noise is most marked in the cer scene, as was found previously. ${ }^{6}$ There is a gradual decrease in quality with increasing noise; detection greatly decreases with noise in the cer scene where the background noise is quite noticeable.

We also see that the effect of contrast is relatively small; the lowest contrast gives the lowest quality, but detection seems to be best at intermediate contrast values. This may be caused by the fact that very low contrast hampers detection, but high contrast in combination with noise also lowers the perceptibility of small details.

The effect of blur is greater, at least for the quality scores. We see that all quality scores drop by approximately 15 times the standard error on passing from the lowest to the highest amount of blur. This is in agreement with remarks made by the subjects, who complained about the blurred images.

Detection is affected to some extent by the interaction between contrast and blur; the effect of blur is most apparent at the lowest contrast level. For quality, on the other hand, blur and contrast have independent effects.

The interaction between contrast and noise that was noticed previously ${ }^{6}$ (lower contrast is preferred when there is more noise) was also found in the current study. This can be seen in the top row of Fig 6: the curves corresponding to the highest contrast are the highest ones when there is no noise, but they drop below the others as the noise increases.

Experiment 2. With the confidence rating, it was less straightforward to arrive at percentages than it was in the first experiment. The following reasoning was applied. If a subject does not see the detail at all in a stimulus and consequently answers "?," then he would answer "A" in $50 \%$ of the trials in a $2 \mathrm{AFC}$ experiment. If a subject answers "A, sure," we assume that he would answer "A" in $100 \%$ of the trials in the 2AFC experiment. The answer " $\mathrm{A}$, not sure" is
Table 5. ANOVA Significant Factors for Experiment 2

\begin{tabular}{lc}
\hline \multicolumn{1}{c}{ Factor } & \% Explained Variance \\
\hline Scene & 24.0 \\
Scene/noise & 22.7 \\
Noise & 13.9 \\
Blur & 9.3 \\
Contrast & 4.2 \\
Noise/contrast & 4.0 \\
Noise/contrast/blur & 3.6 \\
Contrast/blur & 3.4 \\
Error & 0.7 \\
\hline
\end{tabular}

The dependent variable is percentage correct.

then treated as an in-between case and arbitrarily mapped to $75 \%$ correct: the subject would say " $A$ " three of four times in a 2 AFC experiment. It is then possible to compute percentages when the task is repeated. Thus if a subject says "A, sure" once and "A, not sure" once, then answer "A" will be given in $(100 \%+75 \%) / 2=87.5 \%$ of the trials. This gives a mapping of answers with confidence ratings to percentages of correct answers.

The results of the quality rating and detection task were dealt with as in the previous experiment, but here, type of subjects is an extra independent factor in the ANOVA. Thus the subjects are averaged within each group (nonexperts, application specialists, and radiologists). The results are given in Tables 5 and 6 .

Figures 7 and 8 show the results in plots similar to those for experiment 1 . The SEM (after averaging over the four subjects in each group) is less than 0.1 for the detection scores; the $\mathrm{z}$-corrected quality scores are again shown on a scale with unit SEM.

All three types of subjects show very similar results, both for detection and for quality. This also holds for the individuals within each group, as follows from the small SEM. It is seen, too, that the results in this experiment are quite similar to those of experiment 1 . As before, scene has a large effect on detection, but not on quality (although the reservation concerning the use of different quality scales for different

Table 6. ANOVA Significant Factors for Experiment 2

\begin{tabular}{lc}
\hline Factor & \% Explained Variance \\
\hline Blur & 61.2 \\
Contrast & 21.3 \\
Error & 0.5 \\
\hline
\end{tabular}

The dependent variable is $z$-corrected quality. 

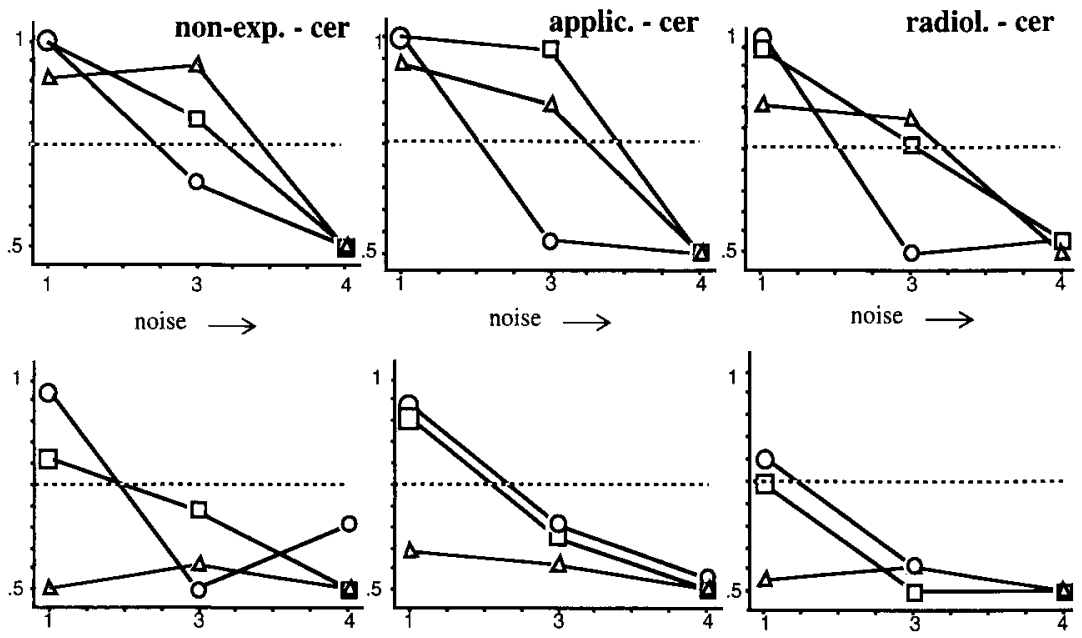

Fig 7. Percentage correct for three types of viewers (left-hand column, nonexperts; middle column, application specialists; right-hand column, radiologists). The two top rows are for the cer scene and the two bottom rows are for kid. The odd and even rows show results for the lowest and highest blur level, respectively. The noise level is indicated on the $X$-axis (see Table 1). The $Y$-axis shows the fraction of correct answers, averaged over the subjects. The dashed line shows the $75 \%$ level. The contrast level is encoded in the type of data points: $(0), 255$; $(\square)$, 200; $\{\triangle\}, 100$.
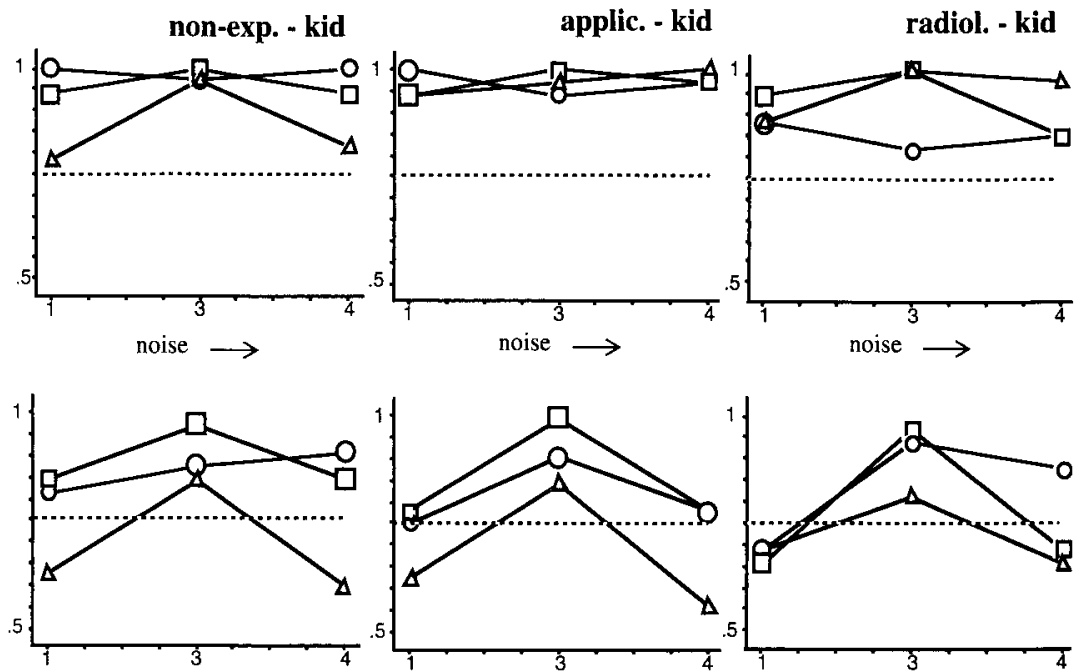

scenes holds again in this case). Noise mainly affects detection in the case of the cer scene, but it also has a slight effect on the perceived quality of both scenes. Contrast affects the quality scores in the sense that the lowest contrast gives the lowest scores in all cases. This effect is most prominent for the application specialists. Blur has a marked effect on the perceived quality; again, this is most striking for the application specialists. Detection also decreases with increasing blur, but mostly when the contrast is low.

\section{COMPARISON WITH MODEL}

In this section, we reconsider our results for the detection experiments and compare them with a model. The main results of the detection experiment can already be explained from Fig 4, showing the profile of the detail for the cer scene at various extreme parameter settings. At least these graphs-and similar ones for the other scenes and other parameter settings-can explain why the detail cannot be detected in certain cases. As an example, for scene cer at the highest noise level, with high contrast and low blur, it is seen that the detail disappears in the noise, even at the level of pixel values. Consequently, because the detail is lost in the data itself, it cannot be visible.

For the cases in which the detail is still present in the data at the pixel-value level, we need a model to predict the visibility of the detail. Several models are known, based on statistical decision theory and/or verified by experiments with very simple stimuli (diskshaped signals on a uniform background). The 

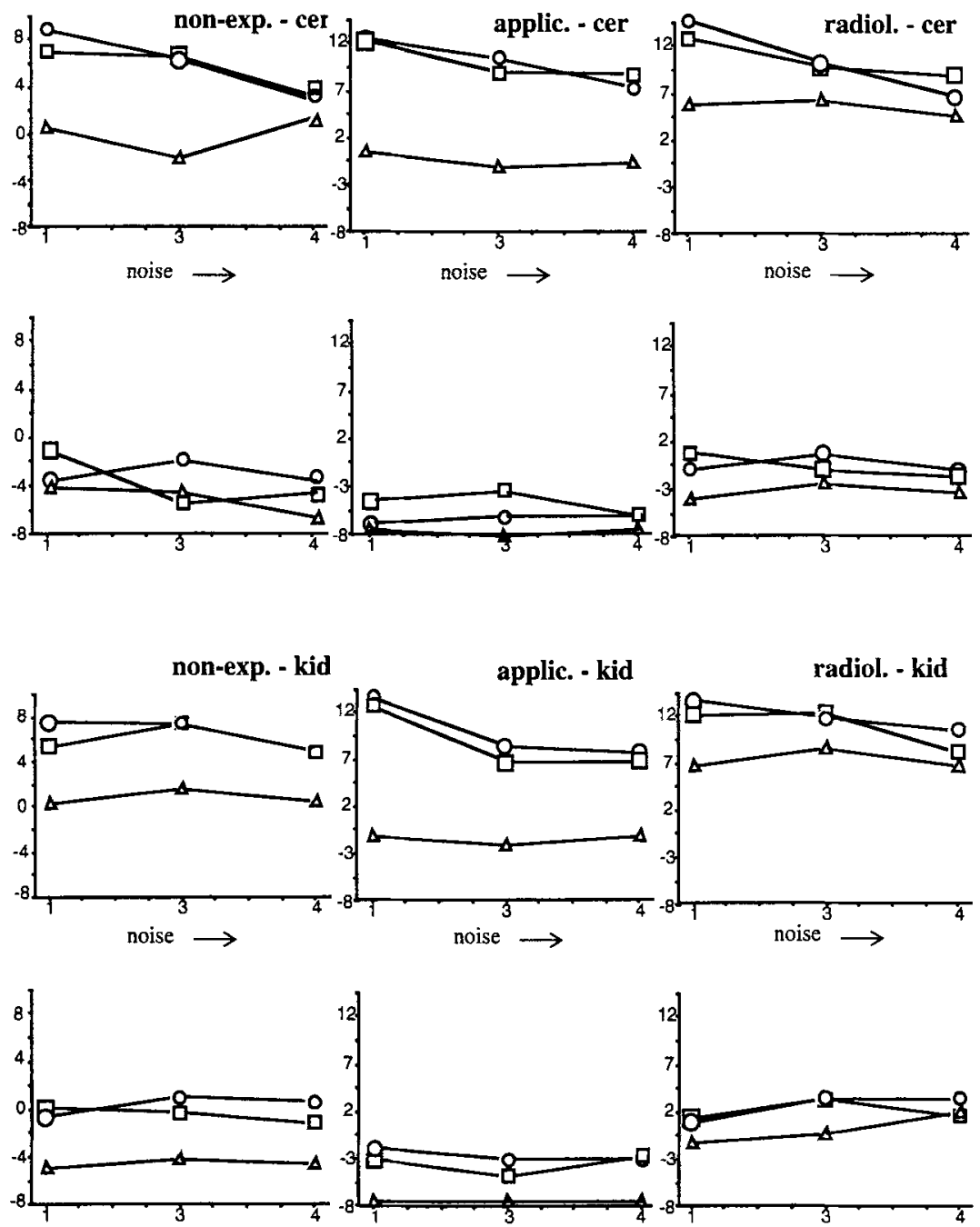

Fig 8. Quality for three types of viewers. The order of the graphs and the labeling of curves is the same as in Fig 7 . The $Y$-axis shows the z-transformed quality scores averaged over the subjects within each group, where the unit equals the SEM.

reader is referred to the Rose model and its extensions. ${ }^{17-19}$ In its basic form, this model says that the amount of contrast needed to make a signal just discernible from its background is linearly related to the amount of noise:

$$
\Delta \mathrm{L}_{\mathrm{T}}=\mathrm{k} \cdot \sigma_{\mathrm{N}}(\mathrm{L}) \sqrt{(\mathrm{n} / 2)}
$$

where $\Delta L_{T}$ is the just-noticeable difference in the luminance domain, $\sigma_{N}(L)$ is the standard deviation of the noise per image pixel, also in terms of luminance, and $n$ is the number of pixels in the object to be detected. In other words, the signal-to-noise ratio (SNR) has to exceed a number, $k$. The factor $k$ ranges from 3 to 5 , as found in most experiments; this depends on the size of the object to be detected, on the percentage chosen for the threshold level, and on various other factors in the experimental set up. In using the above equation, we assume that the internal noise is negligible compared with the considerable amounts of noise added to the stimuli.

Extensions of this model take into account the spectral contents of the signal and the noise, plus the spatial frequency-response function of the human visual system. This can be done for simple stimuli, but in our case, where both the object (vessel) and its background are natural, the spectral decomposition would be irregular and anisotropic, making it very difficult to interpret the contribution of each frequency. On the other hand, we have seen that blur has no significant effect on the detection (in experiment 1), so that the frequency content of the detail does not appear to play a significant role in the detection model. The same observation 
was made for bar detection, ${ }^{20}$ where it was shown that detection of bars in noise yielded the same results for bars with sharp and fuzzy edges, at least for the bar (ie, vessel) width as used in our experiments (a visual angle of around $1 \mathrm{mrad}$ ). The same experiments show that bar width has relatively little effect on the detection of the bars in the range of widths used by us. ${ }^{21)}$ In other words, the object size as expressed in the number of pixels, $n$, does not appear to be a good measure of the detectability of bars. For lack of a better one, we will use this measure all the same. In our case, the vessel covers about 10 to 20 pixels, depending on how the border of the vessel is defined. We will take $n=10$.

Equation 1 can be rewritten in terms of pixel values, ${ }^{21}$ with $\Delta L_{T}$ replaced by $\Delta g$ (the difference in gray value between signal and background), and $\sigma_{N}(L)$ by $\sigma_{N}(g)$ : the standard deviation of the noise per pixel in terms of gray values. This transformation is permissible if $\Delta L / L$ is approximately linear with $\Delta g$ or, equivalently, if $\log (L)$ is linear with $g .{ }^{21}$ Though this is not true for the whole gray-value range in our case, the approximation is sufficiently close in the gray-value ranges of the vessel to be detected and its immediate background for each of the scenes.

For each configuration of object (vessel) and background, the SNR can be computed as

$$
\mathrm{SNR}=\sqrt{(\mathrm{n} / 2)}\left(\mathrm{g}_{\mathrm{b}}-\mathrm{g}_{\mathrm{s}}\right) / \sigma_{\mathrm{N}}(\mathrm{g}),
$$

where $g_{s}$ is the minimum gray value in the vessel and $g_{b}$ the average gray value of the background around the detail. $\sigma_{N}(g)$ is computed as the standard deviation of the gray values in a square area of $30 \times 30$ pixels in the immediate neighborhood of the vessel. The SNR was computed for every contrast-noise combination for the case without blur. The blurred images have been excluded from the analysis because, as argued above, the model of equation 1 does not take into account the effect of spatial frequency. Figure 9 shows the detection probability found in experiment 1 as a function of the SNR for all contrast-noise combinations and all scenes.

The plot shows that all cases with an SNR higher than 5.2 are above the threshold of detectability if the threshold is set at $75 \%$. This

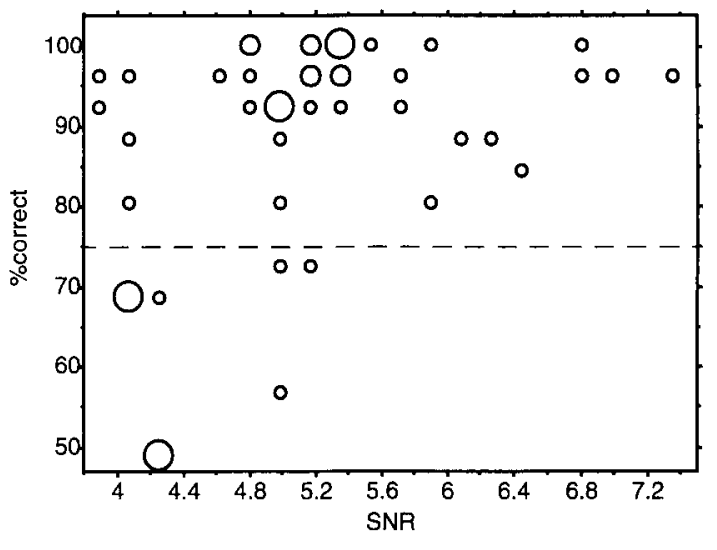

Fig 9. Percentage correct (averaged over all subjects in experiment 1) as a function of SNR. The plot shows a scattergram of all data points with blur level 0 . The larger the circles, the more data points correspond to that combination of SNR and percentage correct. The dashed line represents the threshold level at $75 \%$.

is in reasonable correspondence with the value of $k$ in the Rose model (equation 1), which is usually found to be in the range of 3 to 5 . However, note that values around 5 are mostly obtained for $100 \%$ detection, whereas the value of 3 holds for $50 \%$ detection. Our detection threshold of $75 \%$ correct answers in the $2 \mathrm{AFC}$ experiment should be compared with a threshold of $50 \%$ visibility, so that the value of $k$ found here is relatively high. However, the plot also shows that there are quite a few instances where detection is above threshold if the SNR is lower than 5.2. If we differentiate the data points in Fig 9 with respect to scene, it turns out that these well-detected points with low SNR occur almost exclusively in the kid scene. If the results of the cer scene alone are plotted, we find a better fit to the model, as seen in Fig 10.

It appears that subtracted images, in which only a few different gray values occur, fit the model better than contrast images, where the object (vessel) should be detected against a more complex background. A similar observation was made earlier. ${ }^{22}$

A final remark about the appropriateness of the model for these detection results concerns the fact that the results were obtained with frozen noise, meaning that a single noise pattern was used for every scene and noise level. Thus, the visibility of details at a given noise level could be influenced by the actual instance of the stochastic noise process. 


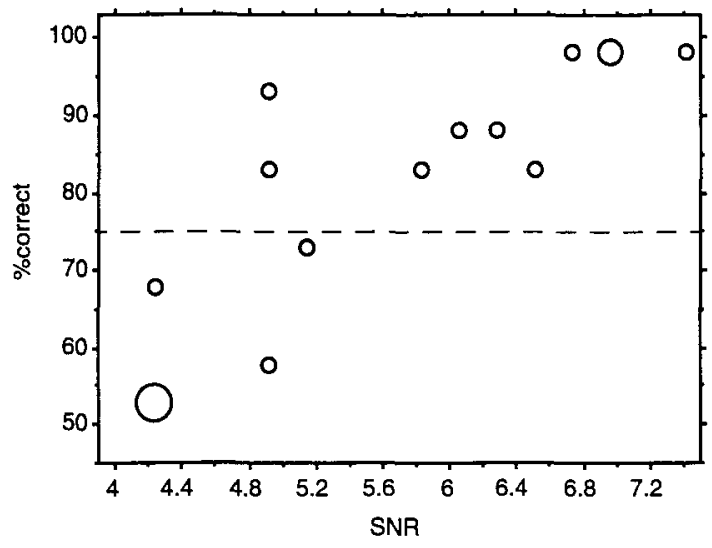

Fig 10. Percentage correct laveraged over all subjects in experiment 1) as a function of SNR: data points for the cer scene and blur level 0 only.

\section{CONCLUSION AND DISCUSSION}

In this final section, the most important findings are summarized and discussed. To start with, we note that the two experiments give quite comparable results if we restrict ourselves to the results of the nonexperts. It is not obvious that both quality scores and detection percentages should accord when they are measured in two different experiments.

As far as the quality scores are concerned, it is known ${ }^{23}$ that the distribution of stimuli used in an experiment may influence a subject's ratings. So, if there are relatively many good stimuli in the set, an average stimulus can get a lower score than if there are relatively many bad stimuli. Our second experiment had an asymmetric stimulus distribution because one of the levels of each factor was excluded. Nevertheless, the quality scores were the same for the experiments with the symmetric and asymmetric distributions. As for the detection, the similarity of the results means that a 2 AFC experiment with five repetitions gives the same result as a $2 \mathrm{AFC}$ experiment with confidence rating and two repetitions.

Furthermore, we have seen that the two different types of measurement (for appreciation- and performance-oriented quality) give quite different results. Thus the well-known approach of measuring how well certain details can be detected, either by phantom measurements or clinical studies, does not guarantee that images optimized in that way can be consid- ered to have high quality. Quality is a combination of appreciation and performance, so it is necessary to measure both types of quality when evaluating a given imaging system or parameter setting. The difference between the two types is reflected in the following findings. Appreciation (the quality score) is mostly affected by blur, a little by contrast (the highest contrast is optimum except when there is much noise), and even less by noise. Detection (the percentage of correct answers), on the other hand, is greatly affected by noise for the subtraction image used in these investigations, and it is affected to some extent by blur when both contrast and noise levels are low.

Another important finding is the fact that nonexperts, application specialists, and radiologists all give the same results for the two tasks used in our experiments-quality scoring and detection of a vessel-and for the scenes and types of processing considered. This follows from comparing the average results of the three groups of subjects and noting that differences between subjects within individual groups are small. A similar result was found for the detection of masses in mammograms. ${ }^{24}$ It would be unwise to conclude from this that it is not necessary to involve radiologists in the evaluation of an imaging device, because we cannot be sure that the detection task used in these experiments is sufficiently similar to the actual tasks of the radiologists (even though the choice of detail added in these experiments was based on interviews with radiologists). If experiments like these are to give reliable estimates of performance-oriented quality, then the subject's task for the performance-oriented quality should be relevant. This task depends on the application, ie, it is necessary to know the type of detail the radiologist wants to see in every kind of image (such as a vessel of minimum diameter and a minimum concentration of contrast agent at a minimum dose level). Once this type of relevant detail is known, an artificial detail having these characteristics (density, size, and shape) can be added to an image for a detection experiment.

Finally, it was shown that the visibility of complex details like the ones used here may be predicted from a model, although the model applied in this study proved to be of limited use: 
it only gave fairly reliable results for subtraction images without blur when the external noise was much stronger than the internal noise. An extension of this model to include the visibility of the different spatial frequencies could give a better prediction, but this has not been investigated in our research.

\section{ACKNOWLEDGMENT}

We thank the staff members of Philips Medical Systems who provided the images and took part in the discussion about this work, especially Herman Stegehuis, and all who acted as subjects, namely my IPO colleagues, the PMSN application experts Jantine Looden, Marjolein van Osta, Hans Aerts and Erik von Reth, and the radiologists Drs H. Van Heesewijk, T. Overtoom, D. Piers, and J. Zwemmer.

\section{REFERENCES}

1. Evans AL: The evaluation of medical images. Medical Physics Handbook 10. Bristol, UK, Adam Hilger, 1981

2. Kanamori $H$, Matsumoto $M$ : The information spec trum as a measure of radiographic image quality and system performance. Phys Med Biol 29:303-313, 1984

3. Wagner RF, Brown DG: Unified SNR analysis of medical imaging systems. Phys Med Biol 30:489-518, 1985

4. Chesters MS: Human visual perception and ROC methodology in medical imaging. Phys Med Biol 37:1433 1476,1992

5. Kundel $\mathrm{H}$ : Visual perception and image display terminals. Radiol Clin N Am 24:69-78, 1986

6. Van Overveld WMCJ: The effect of gamma and noise on perceived quality of $\mathrm{x}$-ray images. Eindhoven, the Netherlands, IPO, Report No. 952, 1993

7. Van Overveld WMCJ: The effect of gamma on subjective quality and contrast of $x$-ray images. Proc SPIE 2166:96104,1994

8. Roufs JAJ, Goossens AMJ: The effect of gamma on perceived image quality. 1988 Proceedings of the International Display Conference, San Diego, CA, 1988, pp 27-31

9. Roufs JAJ: Brightness contrast and sharpness, interactive factors in perceptual image quality. Proc SPIE 1077:6672,1989

10. MacMahon H, Metz CE, Doi K, et al: Digital chest radiography: Effect on diagnostic accuracy of hard copy, conventional video, and reversed gray scale video display formats. Radiology 168:669-673, 1988

11. Cosman PC, Gray RM, Ohlsen RA: Evaluating quality of compressed medical images: SNR, subjective rating, and diagnostic quality. Proc IEEE 82:919-932, 1994

12. Roufs JAJ, Boschman MC: Visual comfort and performance, in Cronley-Dillon JR, Roufs JAJ (eds): Vision and Visual Dysfunction, vol 15. The Man-Machine Interface. New York, NY, MacMillan, 1991, pp 24-40

13. Van Overveld WMCJ: A study on perceived quality of digital x-ray images based on interviews with radiologists in The Netherlands, Switzerland and Great Britain. Eindhoven, The Netherlands, IPO Report No. 861, 1992

14. Van Overveld WMCJ: A study on X-ray image quality based on interviews with radiologists in the USA. Eindhoven, the Netherlands, IPO, Report No. 921, 1993

15. Pizer SM: Intensity mappings to linearize display devices. Comput Graphics Image Process 17:262-268, 1981

16. Chatfield C: Statistics for Technology (ed 3). London, UK, Chapman and Hall, 1989, section 11.6

17. Ji T, Roehrig H, Blume H, Seeley G, et al: Physical and psychophysical evaluation of CRT noise performance. Proc SPIE 1444:136-150, 1991

18. Rose A: The sensitivity performance of the eye on an absolute scale. J Opt Soc Am 38:196-208, 1948

19. De Vries H: The quantum character of light and its bearing upon the threshold of vision, differential sensitivity and visual acuity of the eye, Physica, 10:553-564, 1943

20. Burgess AE, Wagner RF, Jennings RJ: Human signal detection performance for noisy medical images, in: Proceedings International Workshop on Physics and Engineering in Medical Images, IEEE, 1982, pp 99-107

21. Ohara K, Chan HP, Doi K, et al: Investigation of basic imaging properties in digital radiography. 8 . Detection of simulated low-contrast objects in digital subtraction angiographic images. Med Phys 13:304-311, 1986

22. Ohara K, Doi K, Metz CE, et al: Investigation of basic imaging properties in digital radiography. 13. Effect of simple structured noise on the detectability of simulated stenotic lesions. Med Phys 16:14-21, 1989

23. Mellers BA, Birnbaum MH: Loci of contextual effects in judgment. J Exp Psychol Hum Percept Perform 8:582-601, 1982

24. Puff DT, Pisano ED, Muller KE, et al: A method for determination of optimal image enhancement for the detection of mammographic abnormalities. (submitted) 